\title{
Recruitment of Proteins Assessed with Quantitative Live Cell Fluorescence Imaging and Significance Estimates: Example of Endocytic Vesicle Formation
}

\author{
David Perrais ${ }^{1}$, Marcus Taylor ${ }^{2}$, Damien Jullié $^{1} \&$ Christien Merrifield ${ }^{2}$ \\ ${ }^{1}$ Physiologie Cellulaire de la Synapse, CNRS UMR5091 \& University Bordeaux 2, \\ Bordeaux, France \\ ${ }^{2}$ Department of Cell Biology, LMB MRC, Cambridge, United Kingdom
}

Live cell imaging of fluorescently labeled proteins provides a powerful method to investigate the dynamics and role of a protein in a cellular process. However, most imaging studies lack a quantitative analysis of the recruitment kinetics of the given protein and an evaluation of the significance of this recruitment. Here we have used quantitative analysis to study the recruitment of proteins to nascent endocytic vesicles. We have developed a protocol to detect the formation of clathrin coated vesicles (CCVs) from the plasma membrane of Swiss 3T3 cells (Merrifield et al., 2005) or hippocampal neurons. Briefly, cells were transfected with the transferrin receptor, which is constitutively internalized through a clathrin dependent pathway, fused to phluorin (TfR-pHl), a green fluorescent protein which is not fluorescent at pH 5.5 (Miesenbock et al., 1998). The part of the cell adhering to the glass coverslip was imaged with total internal reflection fluorescence (TIRF) microscopy (Figure 1A). By alternating the extracellular $\mathrm{pH}$ between 7.4 and 5.5 it is possible to detect the formation of CCVs with a time resolution of $2 \mathrm{~s}$ (Figure 1B). Several hundred events are easily detected in single cells in 10 min recordings.

We took advantage of the large number of detected events and the temporal precision of the assay to measure fluorescence signals during CCV formation. Events were detected with an automated procedure using object segmentation and tracking (Racine et al., 2006), and trajectories further selected with minimal criteria for bona fide scission events. The TfR-pHl signal at pH 5.5 (TfR5) rose abruptly and decayed in 40 s. At pH 7.4, the signal peaked on average $\sim 10 \mathrm{~s}$ before vesicle creation and decayed (Figure 1D). To evaluate the degree of TfR enrichment at the site of CCV formation, we shifted events in random directions and distances and quantified signals, generating a randomized dataset. We can thus define $95 \%$ confidence intervals for specific enrichment at the site of scission (Figure 1F). Indeed, TfR5 was within random values before $\mathrm{CCV}$ detection.

To evaluate the involvement of associated proteins, we have cotransfected, together with TfR-pHl, RFP fusion proteins. We have performed the same analysis as described before to the RFP signal, with appropriate image registration and bleed-through correction. We have determined the kinetics and degree of protein enrichment at the site of scission proteins involved in CCV formation, such as dynamin, and compared its dynamics in the two cell types studied (3T3 cells and neurones). We will present a detailed analysis of these recruitment parameters in the forthcoming communication. Finally, for 3T3 cells, this analysis has been extended to 34 endocytic proteins defining the dynamic organization of the protein network involved in clathrin mediated endocytosis in live cells (Taylor et al., submitted). 


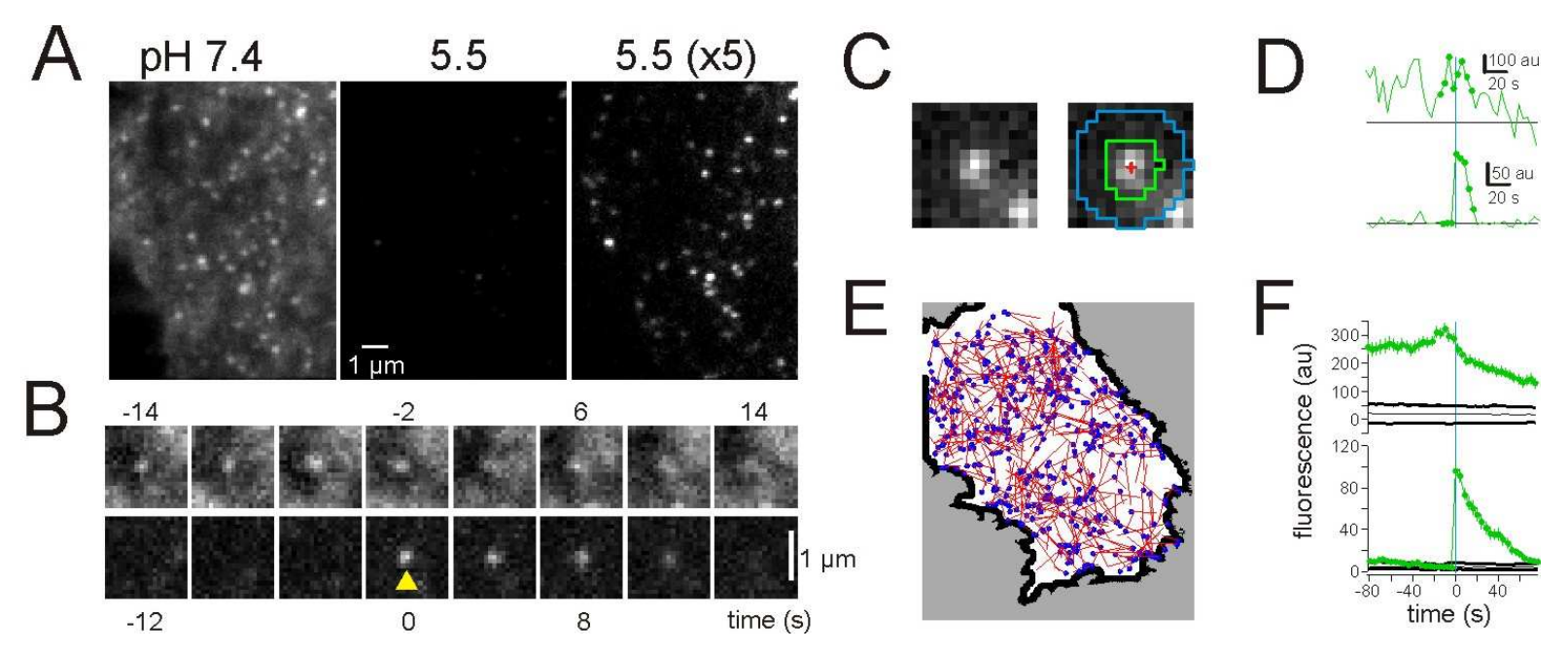

Figure 1: Detection of $\mathrm{CCV}$ formation in $3 \mathrm{T3}$ cells and signal quantification. A. Images of a portion of a 3T3 cell viewed with TIRF microscopy at normal extracellular $\mathrm{pH}$ (7.4) and $2 \mathrm{~s}$ later at $\mathrm{pH}$ 5.5. Left and middle images shown at the same contrast. Right image is shown at $5 \mathrm{x}$ contrast increase to reveal the visible intracellular objects. B, Successive images taken 2 $\mathrm{s}$ apart at $\mathrm{pH} 7.4$ (top) and 5.5 (bottom). At time 0, a CCV is detected at $\mathrm{pH} 5.5$ (yellow arrowhead). C, Illustration of signal quantification. Red cross, center of mass of detected object; signal is average of a circle (radius 3 pixels) around center (green) - average of an annulus (internal radius 3 pixels, external radius 6 pixels; blue). D, Quantification of the signals associated with the event shown in B. E, Randomization procedure. Scission events are shown as blue dots. Red lines show random shifts used to determine confidence intervals (see F). White area shows the center part of the recorded cell, and black area the cell edges. F, average of 154 events aligned to the time of CCV detection (green lines) together with 95\% confidence intervals determined with 200 random shifts (black lines).

\section{References}

Merrifield, C. J., Perrais, D., and Zenisek, D. (2005). Coupling between Clathrin-Coated-Pit Invagination, Cortactin Recruitment, and Membrane Scission Observed in Live Cells. Cell 121, 593-606.

Miesenbock, G., De Angelis, D. A., and Rothman, J. E. (1998). Visualizing secretion and synaptic transmission with $\mathrm{pH}$-sensitive green fluorescent proteins. Nature 394, 192-195.

Racine, V., Hertzog, A., Jouanneau, J., Salamero, J., Kervrann, C., and Sibarita, J. B. (2006). Multipletarget tracking of 3D fluorescent objects based on simulated annealing. Paper presented at: IEEE International Symposium on Biomedical Imaging. 